\title{
Additive moulding \& manufacturing at VMR
}

Thomas Viebrans, VMR GmbH \& Co. KG

This manuscript is not available according to publishing restriction.

Thank you for your understanding.

(C) Springer Fachmedien Wiesbaden GmbH 2017

M. Bargende, H.-C. Reuss, J. Wiedemann (Hrsg.), 17. Internationales Stuttgarter Symposium, Proceedings, DOI 10.1007/978-3-658-16988-6_41 